Research report

\title{
Allured or alarmed: Counteractive control responses to food temptations in the brain
}

\author{
Paul A.M. Smeets ${ }^{\mathrm{a}, \mathrm{c}, *, 1}$, Floor M. Kroese ${ }^{\mathrm{b}, 1}$, Catharine Evers ${ }^{\mathrm{b}}$, Denise T.D. de Ridder ${ }^{\mathrm{b}}$ \\ a Image Sciences Institute, UMC Utrecht, Heidelberglaan 100, 3584 CX, Utrecht, The Netherlands \\ ${ }^{\mathrm{b}}$ Clinical and Health Psychology, Utrecht University, Heidelberglaan 1, 3584 CS, Utrecht, The Netherlands \\ ${ }^{c}$ Division of Human Nutrition, Wageningen University, Wageningen, The Netherlands
}

\section{H I G H L I G H T S}

- We measured brain responses to food temptations in successful dieters.

- Food temptations activate not only reward but also self-control areas in dieters.

- Diet importance predicts food-induced brain activation in self-regulation areas.

- Our neuroimaging findings support counteractive control theory.

\section{A R T I C L E I N F O}

\section{Article history:}

Received 13 March 2013

Received in revised form 26 March 2013

Accepted 31 March 2013

Available online 8 April 2013

\section{Keywords:}

Food temptation

Functional MRI

Counteractive control theory

\begin{abstract}
A B S T R A C T
Typically, it is believed that palatable, high caloric foods signal reward and trigger indulgent responses. However, Counteractive Control Theory suggests that, to the extent that people are concerned about their weight, a confrontation with palatable foods should also trigger 'alarm bell responses' which promote successful self-control. Our study is the first to investigate such counteractive control processes in the brain employing functional magnetic resonance imaging (fMRI) in a sample of successful self-regulators. Indeed, besides the traditional finding that foods elicit heightened attention as witnessed by greater activation of primary visual cortex, we found that viewing palatable foods elicited brain activation in areas associated with self-regulation. Crucially, brain activation in self-regulation areas was related to diet importance. Thus, our results are the first to show that food cues not only evoke hedonic brain responses; in successful self-regulators they also trigger alarm bell responses, which may reflect the neural processes underlying successful self-control.
\end{abstract}

(c) 2013 Elsevier B.V. All rights reserved.

\section{Introduction}

Whereas our ancestors in times of hunters and gatherers faced up to eating as much food as they could possibly find, people in the modern Western food environment face quite a different challenge. The ubiquitous availability of cheap, calorie-dense fast foods is often held responsible for the rapidly rising numbers of people who are overweight or obese [1]. Consequently, many people report to have the intention to watch their weight and limit their food intake in order to prevent weight gain. The conflict between the appeal

Abbreviations: fMRI, functional magnetic resonance imaging; ROI, region of interest; SVC, small volume correction.

* Corresponding author at: Image Sciences Institute, UMC Utrecht, Rm Q0S.459, Heidelberglaan 100, 3584 CX Utrecht, The Netherlands. Tel.: +31 887553172.

E-mail addresses: p.smeets@umcutrecht.nl (P.A.M. Smeets), f.m.kroese@uu.nl (F.M. Kroese), c.evers@uu.nl (C. Evers), d.t.d.deridder@uu.nl (D.T.D. de Ridder).

1 These authors contributed equally to the study. of highly palatable 'unhealthy' foods and weight watching goals represents a typical self-regulation dilemma in which immediate gratification can have negative consequences on the long term.

Since food is a primary reward, it is presumably common sense that people are impulsively drawn toward food cues, especially those that are associated with high energy content, and in particular when people are hungry (e.g., [2]). Counteractive Control Theory, however, suggests that (food) temptations, which trigger indulgence, simultaneously trigger the self-control response that overrides the tendency to indulge [3]. That is, in weight-conscious people unhealthy foods may automatically activate defensive selfregulation mechanisms that serve to protect the weight watching goal and inhibit indulgence [3]. Indeed, empirical evidence for this intriguing notion is accumulating and has been found on cognitive as well as behavioral outcome measures. For example, it was shown that exposure to food temptations increases the mental accessibility of weight watching goals [4], and that participants who were exposed to food temptations subsequently made healthier food choices compared to those in neutral control 
conditions [4,5]. Hence, besides instinctive, reward-driven, approach responses, food cues can also trigger 'alarm bell' responses so as to protect weight watching goals.

Although people's responses toward food cues have gained considerable attention in neuro-imaging studies as well, most research in this area has focused on reward-driven responses toward food. For example, in a recent fMRI study in obese individuals it was found that greater food-cue induced activation in brain regions mediating reward and attentional salience of food cues was associated with less success in a weight-loss program and poorer weight control over a 9-month follow-up period [6].

Conversely, the 'alarm bell'-response has not received particular attention so far. To illustrate, a recent meta-analysis [7] investigated the moderation of brain responses to visual food cues by energy content and hunger, both of which modulate the reward value of food (i.e., food is more rewarding when hunger and/or energy content are high). Whereas the authors argued that dietary restraint (i.e., having adopted a weight watching goal), which in our reasoning would be related to the 'alarm bell' responses in the brain, may indeed serve as a moderator as well, they noted that the number of studies addressing this factor was very small.

The current study aims to address this gap in the literature by specifically focusing on brain activation related to self-regulation in response to tempting (palatable high-caloric) food cues employing fMRI techniques. Although, recently, increasing attention has been devoted to self-regulation in neuro-imaging studies $[8,9]$, no studies have yet reported on counteractive control processes in the brain in response to temptations (but see McCaffery et al. (2009), for a study comparing people who lost weight to lean and obese controls [10]). We hypothesize that in participants with a weight watching goal exposure to food temptations will trigger brain activation in areas related to self-regulation, besides brain areas involved in visual attention and reward processing. We expect these protective self-regulation responses to emerge only to the extent that people have a personally valued weight watching goal. Therefore, we specifically predict that the activation of self-regulation areas in response to food cues will be moderated by the importance attached to the weight watching goal. Our a priori specified regions of interest were based on recent studies of self-regulation in the brain, and include the lateral prefrontal cortex/inferior frontal gyrus and the anterior cingulate cortex, which have been consistently implicated in the exertion of self-control (e.g., $[8,9,11])$.

\section{Materials and methods}

\subsection{Participants}

Participants were thirty healthy, normal-weight, right-handed women with a mean age of 22.1 years $(S D=2.0)$ and a mean body mass index [BMI] of $20.7 \mathrm{~kg} / \mathrm{m}^{2}$, $(\mathrm{SD}=2.0)$. To promote homogeneity, our sample was restricted to females between 18 and 30 years of age, as this is a group known to be concerned about their weight (e.g., [12]). As we were specifically interested in people who successfully deal with food temptations, only participants who had a normal weight (i.e., BMI $18-25 \mathrm{~kg} / \mathrm{m}^{2}$ ) were included. Also, we only included participants who were not high on restraint eating as they are known to have troubled relations with food (e.g., Fedoroff, Polivy, \& Herman, 2003). In addition, we employed common fMRI exclusion criteria (e.g., metal implants in the body, claustrophobia, and pregnancy) and exclusion criteria indicating an altered response to food cues (e.g., metabolic or endocrine disease, gastrointestinal disorders or eating disorders). On average, participants reported to attach moderate importance to dieting $(M=2.7$ on a 5 point scale, $S D=0.8)$. Participants' appetite was moderate before the scan $(M=3.3, \mathrm{SD}=0.9)$, but significantly higher after the $\operatorname{scan}(M=4.1, \mathrm{SD}=0.7 ; t(29)=-5.49, P<.01)$.

\subsection{Study procedures}

Before inclusion in the study, potential participants completed a questionnaire with items on demographic variables (age, length, weight), general health, use of medication, eating disorders [13], restraint eating [14], smoking, alcohol consumption, and contra-indications for fMRI. Eligible participants were requested to refrain from eating and drinking (except water) for at least $3 \mathrm{~h}$ prior to the scanning session.
Upon their arrival, participants indicated the date of their last menstrual period. This was used to assess the current phase of their menstrual cycle, as this affects brain reward responses in general [15] and responses to food images in particular [16,17]. In addition, prior to and after the scan appetite was assessed with 3 items (i.e., "To what extent are you hungry/feeling like a bite/experiencing appetite?" (Cronbach's $\alpha=.91$ and .84 , respectively) that could be answered on a scale from 1 (not at all) to 5 (very much).

Participants were then scanned by use of functional magnetic resonance imaging (fMRI) while viewing images of palatable foods and non-foods (office utensils). After the scan, participants filled out another set of questions including assessments of appetite, and diet goal importance. Diet goal importance was assessed with 2 items (i.e., "To what extent are you concerned about your weight/being slim"; Pearson's $r=.71$; cf. [4]) that could be answered on a scale from 1 (not at all) to 5 (very much)

Written informed consent was obtained from all subjects according to the Declaration of Helsinki (amendment of Seoul, 2008), and the study protocol was approved by the Medical Ethical Committee of the University Medical Center Utrecht, The Netherlands.

\subsection{Task design}

During scanning, subjects alternately viewed $24 \mathrm{~s}$ blocks of palatable food images ( 8 blocks) and non-food images (i.e., office utensils; 8 blocks), interspersed with $8-16$ s rest blocks showing a crosshair (12 s on average). Halfway the task there was a $10 \mathrm{~s}$ break. In the image blocks, 8 images were presented for $2.5 \mathrm{~s}$ each with a $0.5 \mathrm{~s}$ inter-stimulus interval. All pictures were of equal size and displayed the (food) object on a white background. Food pictures were selected to represent foods that are both attractive and 'forbidden' (i.e., fattening), congruent with our definition of temptations [18]. Examples are shown in Supplementary Fig. S.1. A pilot test among 31 female students showed that on average the food pictures were rated as fattening $(M=5.5, \mathrm{SD}=1.2)$, as assessed on a scale ranging from 1 (not at all fattening) to 7 (very fattening) and as rather attractive $(M=5.1, \mathrm{SD}=.9)$, as assessed on a scale from 1 (not at all attractive) to 7 (very attractive). Food pictures were significantly more attractive than office utensil pictures, which were rated as neutral $(M=3.4, S D=1.2$ $P<.01$ ). The tempting nature of the food pictures was confirmed by ratings of random subsamples of these pictures that were obtained from participants in the main study after the scan $(M=4.4, \mathrm{SD}=.8$; on a scale from 1 (not at all tempting) to 7 (very tempting)).

Supplementary data associated with this article can be found, in the online version, at http://dx.doi.org/10.1016/j.bbr.2013.03.041.

\subsection{Data acquisition}

The functional scan was a $\mathrm{T}_{2}{ }^{*}$-weighted gradient echo $2 \mathrm{D}$-echo planar imaging sequence $(64 \times 64$ matrix, repetition time $=1600 \mathrm{~ms}$, echo time $=23 \mathrm{~ms}$, flip angle $=72.5^{\circ}, \quad F O V=208 \times 119 \times 256 \mathrm{~mm}$, SENSE factor $A P=2.4,30$ axial $3.6 \mathrm{~mm}$ slices with $0.4 \mathrm{~mm}$ gap, reconstructed voxel size $=4 \mathrm{~mm} \times 4 \mathrm{~mm} \times 4 \mathrm{~mm}$ ). In one functional run 370 scans were made $(\sim 10 \mathrm{~min})$. In addition to the functional scan, a high resolution $\mathrm{T}_{1}$-weighted anatomical MRI scan was made (3D gradient echo sequence, repetition time $=8.4 \mathrm{~ms}$, echo time $=3.8 \mathrm{~ms}$, flip angle $=8^{\circ}, \quad F O V=288 \mathrm{~mm} \times 288 \mathrm{~mm} \times 175 \mathrm{~mm}, \quad 175$ sagittal slices, voxel size $=1 \mathrm{~mm} \times 1 \mathrm{~mm} \times 1 \mathrm{~mm}$ ). During the functional run, stimuli were presented on a screen with the use of the E-Prime software package.

\subsection{Data processing and analysis}

FMRI data were preprocessed and analyzed with the SPM8 software package (Wellcome Department of Imaging Neuroscience, London, United Kingdom, (http://www.fil.ion.ucl.ac.uk/spm/software/spm8/) in conjunction with the MarsBar toolbox (http://marsbar.sourceforge.net/) run with MATLAB 7.9 (The Mathworks Inc, Natick, MA). The functional volumes of every subject were realigned to the first volume of the first run, globally normalized to Montreal Neurological Institute space (MNI space) retaining $4 \mathrm{~mm} \times 4 \mathrm{~mm} \times 4 \mathrm{~mm}$ voxels, and spatially smoothed with a gaussian kernel of $8 \mathrm{~mm}$ full width at half maximum. A statistical parametric map was generated for every subject by fitting a boxcar function to each time series, convolved with the canonical hemodynamic response function. Data were high-pass filtered with a cutoff of $128 \mathrm{~s}$. Three conditions were modeled: viewing foods, viewing non-foods and the half-way break. For every subject, parameters were estimated for three comparisons (referred to as contrasts); contrast images were calculated for viewing foods $(F)$, viewing non-foods $(N F)$ and for foods $>$ non-foods $(F>N F)$.

First, brain activation by food versus non-food images was assessed using a $t$-test with phase of the menstrual cycle and hunger added as control variables. ${ }^{2}$

\footnotetext{
2 As an additional research question we examined differences between food images presented in color and grayscale versions. Therefore, half of the participants viewed all images in color, whereas the other half viewed grayscale versions of the same images. Post-scan ratings of the images revealed that color and grayscale versions were considered equally attractive. Furthermore, no group differences were found between brain responses to color and grayscale images: there was no
} 
Table 1

Correlations between diet importance and the response to food images. ${ }^{a}$

\begin{tabular}{|c|c|c|c|c|c|c|c|c|}
\hline \multirow[t]{2}{*}{ Region $^{\mathrm{b}}$} & \multirow[t]{2}{*}{ Brodmann area } & \multirow[t]{2}{*}{ Reference coordinate $^{\mathrm{c}}(\mathrm{MNI})$} & \multicolumn{3}{|c|}{ ROI peak coordinate ${ }^{\mathrm{d}}(\mathrm{MNI})$} & \multirow[t]{2}{*}{ Peak Z-score } & \multirow[t]{2}{*}{ Effect size $\mathrm{e}^{\mathrm{e}}$} & \multirow[t]{2}{*}{$r^{\mathrm{f}}$} \\
\hline & & & $x$ & $y$ & $z$ & & & \\
\hline R Calcarine sulcus ${ }^{f}$ & BA18 & $(22,-96,4)$ & 22 & -96 & -2 & 4.5 & 0.82 & 0.67 \\
\hline L Dorsolateral PFC-IFG ${ }^{\mathrm{f}}$ & BA45 & $(-48,22,24)$ & -50 & 32 & 30 & 3.6 & 0.66 & 0.57 \\
\hline R Lateral PFC - anterior insula/IFG & BA47/48 & $(32,28,0)$ & 26 & 32 & 6 & 3.6 & 0.66 & 0.54 \\
\hline R Insula/frontal operculum ${ }^{\mathrm{f}}$ & BA48 & $(42,19,1)$ & 38 & 16 & 10 & 3.0 & 0.55 & 0.44 \\
\hline L Lateral OFC-IFG $^{\mathrm{f}}$ & BA47 & $(-36,31,-3)(-42,41,1)$ & -42 & 32 & -2 & -3.3 & 0.60 & -0.56 \\
\hline
\end{tabular}

a Phase of the menstrual cycle and hunger ratings were included in the model as the first two covariates.

b Abbreviations: IFG: inferior frontal gyrus; L: left hemisphere; OFC: orbitofrontal cortex; PFC: prefrontal cortex, R: right hemisphere.

c Reference peak coordinates obtained from relevant papers [6,9,18,20-22].

d Significant at $P<0.05$ FWE-corrected (SVC, $12 \mathrm{~mm}$ sphere)

e Effect size $=Z$-score $/ \sqrt{ } \mathrm{n}$.

f Pearson correlation coefficient.

g Correlations shown in Fig. 1.

Second, the correlation between diet importance and the response to food images was tested for by entering the $\mathrm{F}>\mathrm{NF}$ contrast images into a $t$-test with hunger and cycle phase as the first two covariates and diet importance as the third. This ensures that effects of diet importance do not overlap with hunger and cycle phase effects. To test our hypothesis that diet importance modulates brain activation by exposure to food cues in visual processing, attention, and self regulation regions, a small volume correction (SVC) analysis was performed in a priori Regions of Interest (ROIs) in the lateral prefrontal and orbitofrontal cortex (inferior frontal gyrus), anterior insula, anterior cingulate, parietal lobule and primary and secondary visual cortex $[7,8,11,19-24]$. First, he whole-brain correlation T-map threshold was set at $P<0.005(T>2.8), k>3$. Subsequently, a priori ROIs were defined as $12 \mathrm{~mm}$ spheres centered on activation peaks (reference peaks) with a Z-score $>3.0$ [25] reported in these previous fMRI studies. Correlations in a priori ROIs were considered significant at $P<0.05$, family-wise error-corrected for multiple comparisons. This approach prevents problems of circularity because of the independence of ROI definitions and correlational analyses [26].

\section{Results}

The average response to food images (food > non-food) is shown in Table A.1 in the appendix. Robust activation of typical reward regions (caudate nucleus, ventral striatum, amygdala) as well as in other regions involved in the regulation of food intake (e.g. hypothalamus) was found, thus corroborating previous findings on the role of reward regions in response to palatable foods.

Diet importance correlated positively with the response to food images ( $F>N F$, i.e., food minus non-food) in the primary visual cortex $(r=0.67)$ and the lateral prefrontal cortex $(r=0.54)$. Furthermore, a negative correlation was found with lateral orbitofrontal cortex activation ( $r=-0.56$ ) (Table 1, Fig. 1$)$. This implies a stronger response of brain areas involved in self-regulation during watching palatable foods in participants who attach more importance to their dieting goals.

Supplementary data associated with this article can be found, in the online version, at http://dx.doi.org/10.1016/j.bbr.2013.03.041.

\section{Discussion}

To our knowledge the current study is the first to investigate counteractive control responses to food cues in the brain as a function of diet importance. Whereas previous research has mostly focused on people's hedonic responses to food cues, based on Counteractive Control Theory [3] we predicted that, to the extent that

significant difference in the response to food images (food vs non-food) between the two groups (two-sample t-test, gray-scale vs color image group with phase of the menstrual cylce and hunger as covariates). A two groups (color: gray/color) $\times$ two image types (food/objects) ANOVA confirmed that there was no significant main effect of image color. In addition, there was no interaction between image color and image type. Therefore, in the current paper we only consider image type (food vs. nonfood). people are concerned about their weight, a confrontation with palatable foods would also activate brain areas that are related to self-regulation. Indeed, besides the traditional finding that foods elicit heightened attention as witnessed by an increase in activity in the primary visual cortex, we found that viewing foods elicited brain activation in a number of areas that are related to selfregulation, and that, crucially, this was related to diet importance.

Our finding that foods elicited elevated (visual) attention is well established in the literature, as a recent meta-analysis also showed that food images elicit stronger activation in visual areas than nonfood images, which was attributed to the higher salience of foods [7]. However, we are the first to report that activation of primary visual cortex correlates with diet importance. Given that even subliminal arousing stimuli induce activation in the primary visual cortex [27], the association between diet importance and increased visual attention to foods may be due to the greater salience of foods for people with a higher diet importance. Importantly, we controlled for hunger such that the greater salience of foods cannot be explained by the motivation to eat. Instead, this finding attests to the suggestion that, to the extent that people are concerned about their weight, foods may indeed trigger 'alarm bell' responses.

More interestingly with respect to the predicted counteractive control responses, we found that diet importance was positively correlated with activation in parts of the inferior frontal gyrus and the anterior insula/frontal operculum. These areas have previously been associated with response inhibition $[23,24,28,29]$ and to a lesser degree with conflict processing [30,31]. For example, the part of the dorsolateral prefrontal cortex (DLPFC) in which food cue activation correlated positively with diet importance in the present study has been found to be activated during active response inhibition in a food-specific go/no-go task [23], and during successful self-control, i.e., choosing healthy foods [29]. This suggests that, in line with the implications derived from Counteractive Control Theory, participants with a higher diet importance may be more likely to adhere to their dieting goal when confronted with palatable foods.

Whereas the above clearly points toward a relation between diet importance and the enhanced activation of self-control supporting areas, our findings with regard to the part of the left OFC in which food cue exposure correlated negatively with diet importance yield a less obvious interpretation. Previous studies yielded inconsistent directions for the role of this part of the left OFC in self-regulation processes $[11,20,23,32]$. Our findings suggest that this area is not supportive of self-control, but rather may stimulate indulgence. This suggestion is corroborated by studies showing that activity in this area has been shown to correlate positively with BMI and, impressively, this response was predictive of weight gain one year later in adolescent girls [20]. On the other hand, however, activation of the left OFC has also been positively associated 

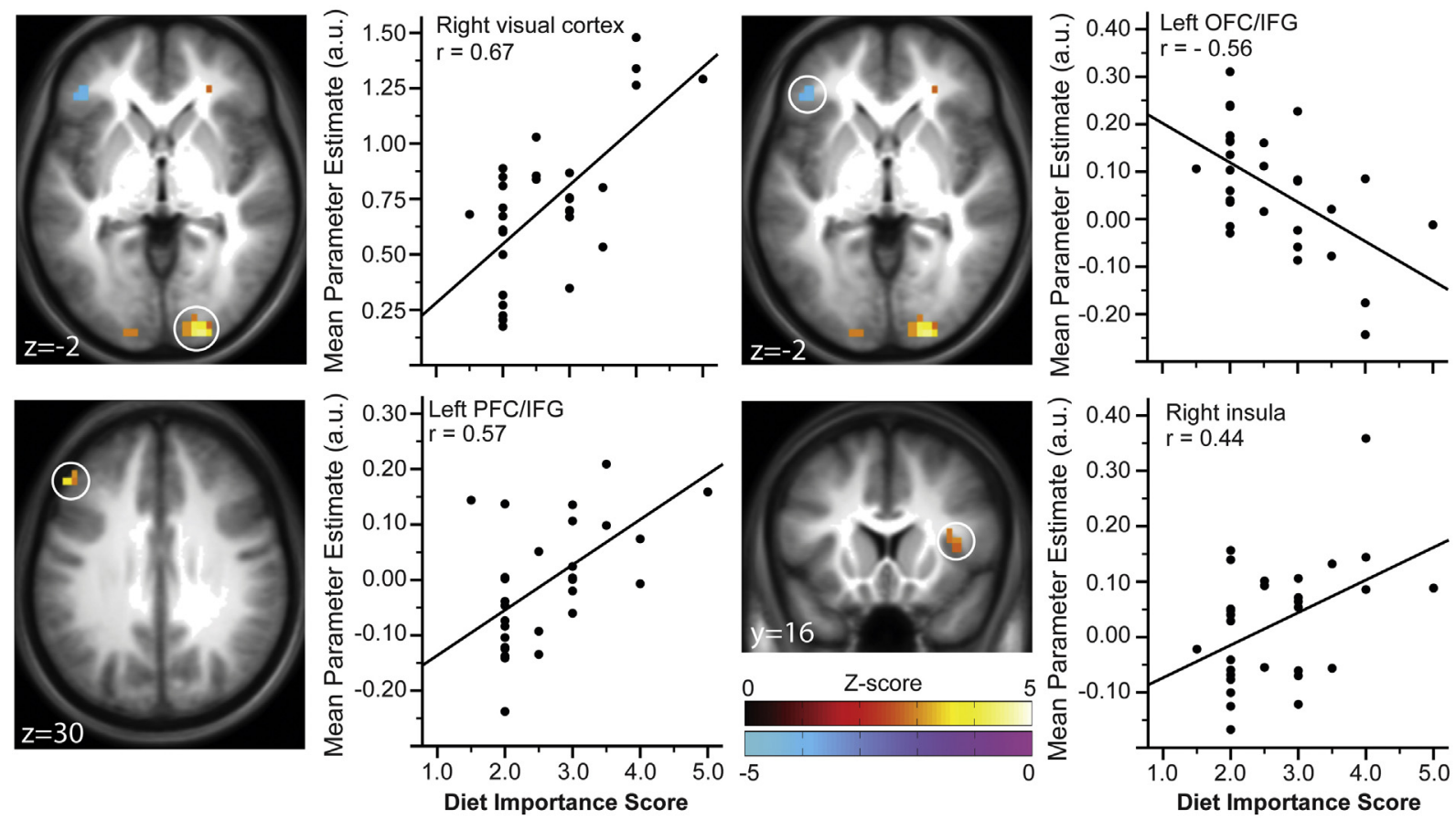

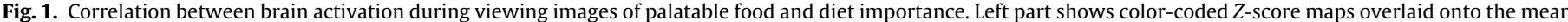

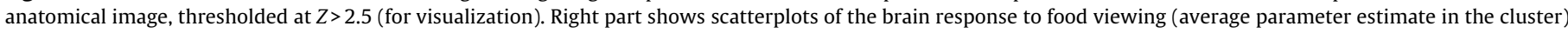

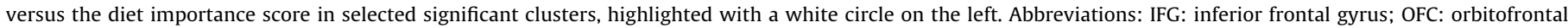
cortex; PFC: prefrontal cortex.

with response inhibition, suggesting that our negative correlation would mean that less inhibition is triggered in people high in diet importance. For example, the left OFC has also been found to be activated during active response inhibition in a food-specific go/nogo task, and this activation was also negatively correlated with BMI [23]. Moreover, a recent study reported stronger activation of this OFC region during making food choices with healthiness in mind, compared to natural food choices [11]. Furthermore, in contrast to the findings reported by Yokum et al. [20], Killgore et al. found a negative correlation between BMI and activation of this region upon exposure to high-calorie food images [32]. In sum, the role of the left OFC in self-regulation processes is yet unclear, and may be dependent on the specific task instructions or outcome measures. In particular, it is important to note that counteractive control processes do not necessarily involve response inhibition but are rather reflective of goal activation upon exposure to temptations.

Altogether, our neuroimaging results point to the existence of adaptive self-regulatory responses to food temptations in people who are concerned about their weight, corroborating findings on cognitive and behavioral outcome measures (e.g., [4,5]). The fact that our findings were obtained while participants were only passively viewing the food images points toward a strong automatic alarm bell response upon exposure to potential threats, as no active behavioral self-regulation was required. Notably, in paradigms that do involve active behavioral self-regulation (e.g., food choice paradigms) we would predict to additionally find activation of typical conflict areas such as the anterior cingulate cortex in weight-concerned individuals. By further elucidating adaptive mechanisms as such, we can increase our knowledge to find new leads to assist successful self-regulation.

Importantly, as the activation of self-control upon confrontation with temptations is reflective of adaptive self-regulation mechanisms, our predictions particularly apply to people who would be classified as successful self-regulators [4,33]. Accordingly, our subjects were all normal-weight and in that sense successful in regulating their food intake [34]. In contrast, it is plausible that our effects would not be found in those who are unsuccessful at resisting food temptations (e.g., overweight or obese people). Rather, a lack of activation of self-regulation areas in response to temptations could explain the failure to limit indulgence. In addition, previous research has provided indications that counteractive control responses are especially likely in response to strong, rather than weak, temptations (e.g., $[18,35])$. Therefore, similar findings may not be expected when participants are confronted with less attractive or less 'forbidden' (i.e., less fattening) foods. Both the effects of weight watching success and temptation strength constitute relevant opportunities for future research.

Another interesting avenue for future research would be to associate brain responses with behavioral outcomes outside of the scanner. For example, related work in a sample of obese participants has shown that weaker responses to food cues in brain reward areas are associated with greater weight loss over a 9month follow-up period [6]. Similarly, we would predict that brain activity in the self-regulation areas discussed above would be related to more favorable behavioral outcomes (e.g., lower consumption of fattening foods) as well. Additionally, our research sets the stage for investigating counteractive control processes in other self-control domains, for example comparing 'alarm bell' responses to smoking-related cues in (ex)smokers who engaged in successful versus unsuccessful quit attempts.

\section{Conclusions}

To conclude, to our knowledge our study is the first to find evidence for counteractive control processes in response to food cues in the brain. We showed that, in people who are watching their weight, food does not only allure them into hedonic responses but also triggers their 'alarm bells'. 


\section{References}

[1] French SA, Story M, Jeffery RW. Environmental influences on eating and physical activity. Annual Review of Public Health 2001;22:309-35.

[2] Pinel JP, Assanand S, Hunger Lehman DR. eating, and ill health. American Psychologist 2000;55(10):1105-16.

[3] Trope Y, Fishbach A. Counteractive self-control in overcoming temptation. Journal of Personality and Social Psychology 2000;79(4):493-506.

[4] Fishbach A, Friedman RS, Kruglanski AW. Leading us not unto temptation: momentary allurements elicit overriding goal activation. Journal of Personality and Social Psychology 2003;84(2):296-309.

[5] Kroese FM, Evers C, de Ridder DT. How chocolate keeps you slim. The effect of food temptations on weight watching goal importance, intentions, and eating behavior. Appetite 2009;53(3):430-3.

[6] Murdaugh DL, Cox JE, Cook III EW, Weller RE. fMRI reactivity to high-calorie food pictures predicts short- and long-term outcome in a weight-loss program. Neuroimage 2012;59(3):2709-21.

[7] van der Laan LN, de Ridder DT, Viergever MA, Smeets PA. The first taste is always with the eyes: a meta-analysis on the neural correlates of processing visual food cues. Neuroimage 2011;55(1):296-303.

[8] Heatherton TF, Wagner DD. Cognitive neuroscience of self-regulation failure. Trends in Cognitive Sciences 2011;15(3):132-9.

[9] Casey BJ, Somerville LH, Gotlib IH, Ayduk O, Franklin NT, Askren MK, et al. Behavioral and neural correlates of delay of gratification 40 years later. Proceedings of the National Academy of Sciences of the United States of America 2011;108(36):14998-5003.

[10] McCaffery JM, Haley AP, Sweet LH, Phelan S, Raynor HA, Del PA, et al. Differential functional magnetic resonance imaging response to food pictures in successful weight-loss maintainers relative to normal-weight and obese controls. American Journal of Clinical Nutrition 2009;90(4):928-34.

[11] Hare TA, Malmaud J, Rangel A. Focusing attention on the health aspects of foods changes value signals in vmPFC and improves dietary choice. Journal of Neuroscience 2011;31(30):11077-87.

[12] Wardle J, Haase AM, Steptoe A. Body image and weight control in young adults: international comparisons in university students from 22 countries. International Journal of Obesity 2006;30(4):644-51.

[13] Stice E, Fisher M, Martinez E. Eating disorder diagnostic scale: additional evidence of reliability and validity. Psychological Assessment 2004;16(1):60-71.

[14] Polivy J, Herman CP, Warsh S. Internal and external components of emotionality in restrained and unrestrained eaters. Journal of Abnormal Psychology 1978;87(5):497-504.

[15] Dreher JC, Schmidt PJ, Kohn P, Furman D, Rubinow D, Berman KF. Menstrual cycle phase modulates reward-related neural function in women. Proceedings of the National Academy of Sciences of the United States of America 2007;104(7):2465-70.

[16] Frank TC, Kim GL, Krzemien A, Van Vugt DA. Effect of menstrual cycle phase on corticolimbic brain activation by visual food cues. Brain Research 2010;1363:81-92.

[17] Alonso-Alonso M, Ziemke F, Magkos F, Barrios FA, Brinkoetter M, Boyd I, et al. Brain responses to food images during the early and late follicular phase of the menstrual cycle in healthy young women: relation to fasting and feeding. American Journal of Clinical Nutrition 2011;94(2):377-84.
[18] Kroese FM, Adriaanse MA, Evers C, de Ridder DT. Instant success: turning temptations into cues for goal-directed behavior. Personality and Social Psychology Bulletin 2011;37(10):1389-97.

[19] Munneke J, Heslenfeld DJ, Theeuwes J. Directing attention to a location in space results in retinotopic activation in primary visual cortex. Brain Research 2008;1222:184-91.

[20] Yokum S, Ng J, Stice E. Attentional bias to food images associated with elevated weight and future weight gain: an FMRI study. Obesity (Silver Spring) 2011;19(9):1775-83.

[21] Fan J, McCandliss BD, Fossella J, Flombaum JI, Posner MI. The activation of attentional networks. Neuroimage 2005;26(2):471-9.

[22] Simmonds DJ, Pekar JJ, Mostofsky SH. Meta-analysis of go/no-go tasks demonstrating that fMRI activation associated with response inhibition is taskdependent. Neuropsychologia 2008;46(1):224-32.

[23] Batterink L, Yokum S, Stice E. Body mass correlates inversely with inhibitory control in response to food among adolescent girls: an fMRI study. Neuroimage 2010;52(4):1696-703.

[24] Hopfinger JB, Buonocore MH, Mangun GR. The neural mechanisms of top-down attentional control. Nature Neuroscience 2000;3(3):284-91.

[25] Beckmann CF, Jenkinson M, Smith SM. General multilevel linear modeling for group analysis in FMRI. Neuroimage 2003;20(2):1052-63.

[26] Poldrack RA, Mumford JA. Independence in ROI analysis: where is the voodoo? Social Cognitive and Affective Neuroscience 2009;4(2):208-13.

[27] Brooks SJ, Savov V, Allzen E, Benedict C, Fredriksson R, Schioth HB. Exposure to subliminal arousing stimuli induces robust activation in the amygdala, hippocampus, anterior cingulate, insular cortex and primary visual cortex: a systematic meta-analysis of fMRI studies. Neuroimage 2012;59(3): 2962-73.

[28] Goldstein M, Brendel G, Tuescher O, Pan H, Epstein J, Beutel M, et al. Neural substrates of the interaction of emotional stimulus processing and motor inhibitory control: an emotional linguistic go/no-go fMRI study. Neuroimage 2007;36(3):1026-40.

[29] Hare TA, Camerer CF, Rangel A. Self-control in decision-making involves modulation of the vmPFC valuation system. Science 2009;324(5927):646-8.

[30] Aarts E, Roelofs A, van TM. Attentional control of task and response in lateral and medial frontal cortex: brain activity and reaction time distributions. Neuropsychologia 2009;47(10):2089-99.

[31] Kemmotsu N, Villalobos ME, Gaffrey MS, Courchesne E, Muller RA. Activity and functional connectivity of inferior frontal cortex associated with response conflict. Brain Research Cognitive Brain Research 2005;24(2):335-42.

[32] Killgore WD, Yurgelun-Todd DA. Body mass predicts orbitofrontal activity during visual presentations of high-calorie foods. Neuroreport 2005;16(8): 859-63.

[33] Papies EK, Stroebe W, Aarts H. Healthy cognition: processes of self-regulatory success in restrained eating. Personality and Social Psychology Bulletin 2008;34(9):1290-300.

[34] Meule A, Papies EK, Kubler A. Differentiating between successful and unsuccessful dieters. Validity and reliability of the perceived self-regulatory success in dieting scale. Appetite 2012;58(3):822-6.

[35] Geyskens K, Dewitte S, Pandelaere M, Warlop L. Tempt me just a little bit more: the effect of prior food temptation actionability on goal activation and consumption. Journal of Consumer Research 2008;35(4):600-10. 